\title{
Index to Volume 12, 2010
}

\author{
5-oxoproline
}

449-52

A

abdominal pain

485-90, 525-6, 538-9

abrasion

389-94

academics

$8-10$

acetaminophen

220-2, 449-52

acute coronary syndrome $\quad 128-34$,

$405-13$

administration $\quad 8-10,11-3,311-9$

adverse drug-related events $331-8$

adverse events

$331-8,421-30$

advocacy

$8-10$

airway

$135-40$

albuterol

$477-84$

alcohol-free drink

224-5

ambulance

appendicitis

arrhythmias

$111-8$

525-6, 538-9

$181-91$

asthma

$339-46,477-84$

atrial fibrillation

atrial flutter

$181-91$

$181-91$

auscultation

63

\section{B}

bag-mask ventilation $\quad 135-40$

brain injuries

bronchiolitis

bupropion

$355-64$

$443-5,477-84$

$158-61$

C

Canadian Emergency Department

Triage and Acuity Scale 45-9

capnography

cardioversion

case report

cast

cellphone

397-404

181-91

449-52

192-200

$365-70$

central venous pressure $\quad 320-4$

cerebral toxoplasmosis $\quad 223,227-8$

cervical strain

chest pain

child
39-44

63
$514-9$ chronic obstructive pulmonary

$$
\text { disease }
$$

clerkship

CLM

closed reduction

coaching

community-acquired

pneumonia

compartment syndrome

compliance

comprehension

computed tomographic

urography

conduite

conscious sedation

consultation

continuity of care

cornea

creatine kinase

creeping eruption

curriculum

cutaneous larva migrans

cyclobenzaprine

cystitis

D

dantrolene

databases

dental emergency

435-42, 457-9

339-46

dental fracture

dental pain

dental trauma

dexamethasone

diagnosis 128-34, 405-13, 528-9

diagnosis classification

diagnostic testing

disaster medicine

disaster planning

discharge

disease management

distal radius (Colles)

fracture

door-to-antibiotic time

driving

500-8

212-9

446

192-200

$520-4$

207-11

453-6

325-30

325-30

377-80

371-6

397-404

325-30

325-30

389-94

64-8

446

212-9, 349-53

446

$39-44$

527,540

192-200

365-70
E

early goal-directed

therapy 150-3, 414-20

ED administration $\quad 50-7$

education

$143-9,347-8$

electrical cardioversion 181-91

emergency 491-9

emergency dental kit $\quad 73$

emergency department 45-9, 181-91, 201-6, 303-10,

311-9, 325-30, 414-20, 485-90

emergency department

overcrowding

$18-26$

emergency medical

$$
\text { services 45-9, 103-10, }
$$

$119-27,128-34$

emergency medical system $\quad 111-8$

emergency medical

technician

$111-8$

emergency medicine $8-10,27-32$, $33-8,143-9,212-9$, 303-10, 331-8, 339-46, 349-53, 421-30, 500-8

emphysema

527,540

endoscopy

$447-8$

énoncé de principe

$371-6$

epidemiology

$339-46,500-8$

epinephrine

$443-5$

error

491-9

evaluation

$33-8,212-9$

extensor digitorum muscle $\quad 453-6$

extraglottic

$135-40$

F

factorial clinical trials

58-61

falls in elderly

$509-13$

feedback

520-4

female

143-9

forearm

453-6

foreign body

447-8

Fournier gangrene

528-9

funding

33-8

G 


\begin{tabular}{|c|c|c|c|c|c|}
\hline \multirow{3}{*}{$\begin{array}{l}\text { grants } \\
\text { grippe humaine }\end{array}$} & $33-8$ & MDMA-related & \multirow[b]{2}{*}{$435-42,457-9$} & \multicolumn{2}{|c|}{$111-8,135-40$} \\
\hline & $16-7$ & hyperpyrexia & & procedural sedation & $397-404$ \\
\hline & & médecine d'urgence & $11-3$ & proparacaine & 389-94 \\
\hline & & medical education & $349-53$ & prophylactic hypothermia & $355-64$ \\
\hline Hamman sion & & & $431-4,520-4$ & propofol & $397-404$ \\
\hline Hamman sign & 63 & medical student & $143-9,212-9$ & pulmonary edema & $224-5$ \\
\hline heac & $223,227-8$ & medication & $331-8$ & vroglutamic acid & $449-52$ \\
\hline head injury & $154-7$ & mémoire & 302 & & \\
\hline headache & $220-2$ & & 301 & & \\
\hline heart arrest & $119-27$ & & $143-9$ & Q & \\
\hline heart failure & $320-4$ & & $449-52$ & quality improvement & $303-10$ \\
\hline $\mathrm{HIV}$ & $223,227-8$ & & 220 & quantitative resuscitation & $150-3$ \\
\hline hookworm & 446 & taire & $\begin{array}{r}220-2 \\
11-3\end{array}$ & & \\
\hline human influenza & $14-5$ & mili & $\begin{array}{r}11-3 \\
446\end{array}$ & $\mathbf{R}$ & \\
\hline nic saline & $477-84$ & $\min$ & $143-9$ & radiog & $128-34$ \\
\hline hypo & $224-5$ & & 509 & rands & $477-84$ \\
\hline hypothermia, induced & $355-64$ & mor & $509-13$ & randomized controlled & \\
\hline & & oskeletal disease & $453-6$ & & $192-200$ \\
\hline & & myocardial infarction & $64-8$ & rapid sequence intubation & $154-7$ \\
\hline bupr & 39-44, 220-2 & & $28-34,405-13$ & al & $447-8$ \\
\hline ilimation tonhni & $92-200$ & & & renal colic & , 377-80 \\
\hline ation de masse & $16-7$ & $\mathbf{N}$ & & reporting & 491-9 \\
\hline . & $69-72$ & nares & $158-61$ & ntation & $11-3$ \\
\hline infe & 527,540 & nasal insufflation & 158 & $33-8,143-9$ & $9,347-8$ \\
\hline ion & $447-8$ & & & residency & $349-53$ \\
\hline inju & $453-6$ & & & faculty & 143-9 \\
\hline severity & $111-8$ & opin & & depression & $397-404$ \\
\hline t care & $18-26$ & oral & & diseases & $500-8$ \\
\hline ledicine & $18-26$ & mes & $\begin{array}{l}509-13 \\
275 \quad 20\end{array}$ & afection & $477-84$ \\
\hline intracranial pressure & $154-7$ & tient referral & $325-30$ & syncytial virus & $477-84$ \\
\hline & & oximetry & 397-404 & tract diseases & $339-46$ \\
\hline & & $\mathbf{P}$ & & ation & $58-61$ \\
\hline jugular v & $320-4$ & pain & 389-94 & & $355-64$ \\
\hline keta & $154-7$ & 103 & $03-10,135-40$ & ed quadriceps tendon & 62,75 \\
\hline knes & 62,75 & afety & $331-8,421-30$ & rural ED & 207-11 \\
\hline knowledge translation & $431-4$ & ped & $69-72$ & & \\
\hline & & & $443-5,514-9$ & & \\
\hline & & pelv & $509-13$ & $365-70$ & $0,491-9$ \\
\hline $\mathbf{L}$ & & pen & $58-61$ & salb & $477-84$ \\
\hline & $50-7$ & perf & $18-26$ & & $224-5$ \\
\hline$g$ methods & $212-9$ & practice pat & atterns $\quad 514-9$ & ship & $347-8$ \\
\hline leng & $50-7$ & ediastinum & 63 & sécl & $371-6$ \\
\hline licor & $224-5$ & pne & $514-9$ & seiz & $158-61$ \\
\hline literature review & $143-9$ & pnet & 63 & $58-61,150-3$, & , 303-10 \\
\hline low-impact trauma & $509-13$ & tion surveillance & $119-27$ & sepsis protocol & $414-20$ \\
\hline & & port & $525-6,538-9$ & $303-10$, & , 414-20 \\
\hline M & & position statement & $365-70$ & $303-10$ & , 414-20 \\
\hline & $449-52$ & postg & $349-53$ & ences & $405-13$ \\
\hline & $14-5$ & e education & $491-9$ & $27-32$, & , 349-53 \\
\hline MDMA & $35-42,457-9$ & potassium & $224-5$ & splint & $192-200$ \\
\hline
\end{tabular}


stroke

supraglottic

systematic review

\section{T}

teaching methods

téléphone cellulaire

tendon rupture

tétanos

tetanus

time to physician
103-10

$135-40$

355-64

$349-53$

371-6

62,75

16-7

14-5, 69-72

50-7
435-42, 457-9

135-40

111-8, 453-6

355-64

traumatic brain injury $45-9,103-10,111-8$

triage

tropical medicine

troponin

446

64-8

$\mathbf{U}$

ultrasonography unstable angina

urethral stone

128-34, 405-13

urolithiasis

$377-80$

urologic intervention

V-W

vaccination

wait time

14-5, 16-7

wheezing
201-6

201-6

$50-7$

$477-84$ 